ANNALES

POLONICI MATHEMATICI

$86.2(2005)$

\title{
Projections onto the spaces of Toeplitz operators
}

\author{
by Marek Ptak (Kraków)
}

\begin{abstract}
Projections onto the spaces of all Toeplitz operators on the $N$-torus and the unit sphere are constructed. The constructions are also extended to generalized Toeplitz operators and applied to show hyperreflexivity results.
\end{abstract}

1. Introduction. Arveson [1] defined a projection from the algebra $B\left(H^{2}(\mathbb{T})\right)$ of all bounded linear operators on the Hardy space on the unit circle onto the space of all Toeplitz operators on $H^{2}(\mathbb{T})$. He used the classical Banach limit. We construct a projection from the algebra $B\left(H^{2}\left(\mathbb{T}^{N}\right)\right)$ of all bounded linear operators on the Hardy space on the $N$-torus onto the space of all Toeplitz operators on $H^{2}\left(\mathbb{T}^{N}\right)$. We use the extension of the Banach limit to $N$-parameter sequences, given in Section 2.

In Section 4 we will use the above projection to show that the subspace of all Toeplitz operators on the $\mathrm{N}$-torus is 2-hyperreflexive (for definition see Section 4). The single variable case was considered in [7].

A natural generalization of the unit circle is not only the $N$-torus but also the unit sphere $\partial \mathbb{B}_{N}$. In Section 5 we construct a projection from the algebra $B\left(H^{2}\left(\partial \mathbb{B}_{N}\right)\right)$ of all bounded linear operators on the Hardy space on the unit sphere onto the space of all Toeplitz operators on $H^{2}\left(\partial \mathbb{B}_{N}\right)$.

In Section 6 we extend the idea of such a projection to generalized Toeplitz operators which were introduced in [10], [11]. We consider both one and multi-variable cases. In Section 7 we give a hyperreflexivity result for generalized Toeplitz operators. The one variable case was considered in $[8]$.

2. Multi-variable Banach limit. There is a functional on all bounded sequences in $\ell^{\infty}$, which to any convergent sequence $\{x(n)\}_{n \in \mathbb{N}}$ assigns its

2000 Mathematics Subject Classification: Primary 47B35; Secondary 47L80, 46B45.

Key words and phrases: Banach limit, Hardy space, Toeplitz operator, reflexive subspace, hyperreflexive subspace, $k$-hyperreflexive subspaces, transitive subspace.

This research was supported by grant No 1 P03A 01627 of KBN, Poland. 
limit (see e.g. [4]). It is called the Banach limit. We extend this idea to multi-variable bounded sequences in $\ell^{\infty}\left(\mathbb{N}^{N}\right)$.

Theorem 2.1. There is a linear functional $\Lambda: \ell^{\infty}\left(\mathbb{N}^{N}, \mathbb{C}\right) \rightarrow \mathbb{C}$ (resp. $\left.\Lambda: \ell^{\infty}\left(\mathbb{N}^{N}, \mathbb{R}\right) \rightarrow \mathbb{R}\right)$ such that

(a) $\|\Lambda\|=1$ and $\Lambda(\mathbf{1})=1$, where $\mathbf{1}$ is the constantly 1 sequence,

(b) if $x \in \ell^{\infty}\left(\mathbb{N}^{N}\right)$ converges, then $\Lambda(x)=\lim _{k \rightarrow \infty, k \in \mathbb{N}^{N}} x(k)$,

(c) if $x \in \ell^{\infty}\left(\mathbb{N}^{N}\right)$ is nonnegative, i.e. $x(k) \geq 0$ for all $k \in \mathbb{N}^{N}$, then $\Lambda(x) \geq 0$,

(d) for any sequence $x \in \ell^{\infty}\left(\mathbb{N}^{N}\right)$ and any $i=1, \ldots, N$, let $x^{(i)}$ denote the sequence $x^{(i)}(k)=x\left(k+e_{i}\right)$, where $e_{i}=(0, \ldots, 1, \ldots, 0)$; then $\Lambda(x)=\Lambda\left(x^{(i)}\right)$.

Proof. First we deal with the case of a real-valued functional, i.e. $\Lambda$ : $\ell^{\infty}\left(\mathbb{N}^{N}, \mathbb{R}\right) \rightarrow \mathbb{R}$. For each $i$, set $\mathcal{M}_{i}=\left\{x-x^{(i)}: x \in \ell^{\infty}\left(\mathbb{N}^{N}, \mathbb{R}\right)\right\}$, where $x^{(i)}$ is defined as in (d). Note that $\mathcal{M}_{i}$ is a linear manifold. Let $\mathcal{M}$ be the subspace spanned by all $\mathcal{M}_{i}, i=1, \ldots, N$. We show first that

$$
d(\mathbf{1}, \mathcal{M})=1,
$$

where $d$ denotes the distance from the sequence 1 to the subspace $\mathcal{M}$. Since $0 \in \mathcal{M}$ we have $d(\mathbf{1}, \mathcal{M}) \leq 1$. Assume that there are $\varepsilon>0$ and $x_{i} \in \mathcal{M}_{i}$ and $\alpha_{i} \in \mathbb{R}$ with $\left\|\alpha_{i} x_{i}\right\|_{\infty} \leq M, i=1, \ldots, N$, such that

$$
\left\|\mathbf{1}-\sum_{i=1}^{N} \alpha_{i}\left(x_{i}-x_{i}^{(i)}\right)\right\|_{\infty}<1-\varepsilon .
$$

In particular, for fixed $n \in \mathbb{N}$, for all $k=\left(k_{1}, \ldots, k_{N}\right) \in \mathbb{N}^{N}$ such that $|k|_{\infty}=\max \left|k_{i}\right| \leq n$, we have

$$
1-\sum_{i=1}^{N} \alpha_{i}\left(x_{i}(k)-x_{i}^{(i)}(k)\right)<1-\varepsilon .
$$

Summation over $k$ gives

Thus

$$
n^{N}-\sum_{i=1}^{N} \alpha_{i}\left(\sum_{\substack{|k|_{\infty} \leq n \\ k_{i}=1}} x_{i}(k)-\sum_{\substack{|k|_{\infty} \leq n \\ k_{i}=n}} x_{i}\left(k+e_{i}\right)\right)<n^{N}-n^{N} \varepsilon .
$$

$$
n^{N} \varepsilon<\sum_{i=1}^{N}\left|\alpha_{i}\right|\left(\sum_{\substack{|k|_{\infty} \leq n \\ k_{i}=1}}\left|x_{i}(k)\right|+\sum_{\substack{|k|_{\infty} \leq n \\ k_{i}=n}}\left|x_{i}\left(k+e_{i}\right)\right|\right) \leq 2 n^{N-1} N M .
$$

Hence $n \varepsilon<2 N M$ and we have a contradiction for $n$ large enough, so (1) follows.

The Hahn-Banach theorem yields a linear functional $\Lambda$ on $\ell^{\infty}\left(\mathbb{N}^{N}, \mathbb{R}\right)$ such that $\Lambda(\mathbf{1})=1, \Lambda(\mathcal{M})=0$ and $\|\Lambda\|=1$. 
To see (b), for a given sequence $x \in \ell^{\infty}\left(\mathbb{N}^{N}, \mathbb{R}\right)$ and for all multi-indices in $\mathbb{N}^{N}$, define by multi-induction the following sequences:

$$
x_{\left(e_{i}\right)}=x^{(i)} \text { and } \quad x_{\left(k+e_{i}\right)}=\left(x_{(k)}\right)^{(i)} .
$$

Note that the definition is correct since $\left(x_{\left(k-e_{i}\right)}\right)^{(j)}=\left(x_{\left(k-e_{j}\right)}\right)^{(i)}$. For fixed $k=\left(k_{1}, \ldots, k_{N}\right) \in \mathbb{N}^{N}$ we have

$$
\begin{aligned}
x_{(k)}-x= & \left(x_{(k)}-x_{\left(k-e_{N}\right)}\right)+\cdots+\left(x_{\left(k_{1}, \ldots, k_{2}, 1\right)}-x_{\left(k_{1}, \ldots, k_{2}, 0\right)}\right) \\
& +\cdots+\left(x_{\left(k_{1}, 0, \ldots, 0\right)}-x_{\left(k_{1}-1,0, \ldots, 0\right)}\right)+\cdots+\left(x_{(1,0, \ldots, 0)}-x\right) .
\end{aligned}
$$

Thus $\Lambda(x)=\Lambda\left(x_{(k)}\right)$. If $x$ is convergent and $\alpha=\lim _{k^{\prime} \rightarrow \infty, k^{\prime} \in \mathbb{N}^{N}} x\left(k^{\prime}\right)$, then

$$
\begin{aligned}
|\Lambda(x)-\alpha| & =\left|\Lambda\left(x_{(k)}-\alpha \cdot \mathbf{1}\right)\right| \leq\left\|x_{(k)}-\alpha \cdot \mathbf{1}\right\|_{\infty} \\
& \leq \sup \left\{\left|x\left(k_{1}^{\prime}, \ldots, k_{N}^{\prime}\right)-\alpha\right|: k_{i}^{\prime}>k_{i} \text { for all } i\right\} .
\end{aligned}
$$

Thus $|\Lambda(x)-\alpha|$ is arbitrarily small and we get $(\mathrm{b})$.

Condition (c) and extension to the case of complex-valued sequences can be shown as in the single variable case (see for example [4]).

3. Projection onto Toeplitz operators on the $N$-torus. Let $\mathbb{T}$ be the unit circle on the complex plane $\mathbb{C}$. Set $L^{2}(\mathbb{T})=L^{2}(\mathbb{T}, m)$ and $L^{\infty}(\mathbb{T})=$ $L^{\infty}(\mathbb{T}, m)$, where $m$ is the normalized Lebesgue measure on $\mathbb{T}$. Let $H^{2}(\mathbb{T})$ be the Hardy space corresponding to $L^{2}(\mathbb{T})$ and let $P_{H^{2}(\mathbb{T})}$ be the orthogonal projection from $L^{2}(\mathbb{T})$ onto $H^{2}(\mathbb{T})$. We denote by $H^{\infty}(\mathbb{T})$ the Hardy space corresponding to $L^{\infty}(\mathbb{T})$, i.e. the space of those functions from $L^{\infty}(\mathbb{T})$ which have an analytic extension to the whole unit disc $\mathbb{D}$.

For each $\varphi \in L^{\infty}(\mathbb{T})$ we define $T_{\varphi} \in B\left(H^{2}(\mathbb{T})\right)$ by $T_{\varphi} f=P_{H^{2}(\mathbb{T})}(\varphi f)$ for $f \in H^{2}(\mathbb{T})$. The operator $T_{\varphi}$ is called a Toeplitz operator with symbol $\varphi$. Let $\mathcal{T}(\mathbb{T})$ denote the space of all Toeplitz operators, and $\mathcal{A}(\mathbb{T})$ the space of Toeplitz operators with symbols from $H^{\infty}(\mathbb{T})$. We have $([6$, Corollary to Problem 194])

$$
\mathcal{T}(\mathbb{T})=\left\{A \in B\left(H^{2}(\mathbb{T})\right): A=T_{z}^{*} A T_{z}\right\}
$$

and by [6, Problem 116],

$$
\mathcal{A}(\mathbb{T})=\left\{A \in B\left(H^{2}(\mathbb{T})\right): A T_{z}=T_{z} A\right\}
$$

Similarly we denote the corresponding spaces on the $N$-torus, $L^{2}\left(\mathbb{T}^{N}\right)$, $L^{\infty}\left(\mathbb{T}^{N}\right), H^{2}\left(\mathbb{T}^{N}\right), H^{\infty}\left(\mathbb{T}^{N}\right)$ and the projection $P_{H^{2}\left(\mathbb{T}^{N}\right)}: L^{2}\left(\mathbb{T}^{N}\right) \rightarrow H^{2}\left(\mathbb{T}^{N}\right)$. For each $\varphi \in L^{\infty}\left(\mathbb{T}^{N}\right)$ we define the Toeplitz operator $T_{\varphi} \in B\left(H^{2}\left(\mathbb{T}^{N}\right)\right)$ by $T_{\varphi} f=P_{H^{2}\left(\mathbb{T}^{N}\right)}(\varphi f)$. We denote by $\mathcal{T}\left(\mathbb{T}^{N}\right)$ the space of all Toeplitz operators and by $\mathcal{A}\left(\mathbb{T}^{N}\right)$ the space of all Toeplitz operators with symbols from $H^{\infty}\left(\mathbb{T}^{N}\right)$. By $T_{z_{i}}, i=1, \ldots, N$, we denote the multiplication operators by the independent variables. Since the operators $T_{z_{i}}$ commute we can set $T_{z^{k}}=T_{z_{1}}^{k_{1}} \cdots T_{z^{N}}^{k_{N}}$ for $k=\left(k_{1}, \ldots, k_{n}\right) \in \mathbb{N}^{N}\left(z^{k}=z_{1}^{k_{1}} \cdots z_{N}^{k_{N}}\right)$. 
Similarly to the one variable case we have the following characterizations (see [9, Proposition 3.3]):

$$
\begin{aligned}
& \mathcal{T}\left(\mathbb{T}^{N}\right)=\left\{A \in B\left(H^{2}\left(\mathbb{T}^{N}\right)\right): A=T_{z_{i}}^{*} A T_{z_{i}}, i=1, \ldots, N\right\}, \\
& \mathcal{A}\left(\mathbb{T}^{N}\right)=\left\{A \in B\left(H^{2}\left(\mathbb{T}^{N}\right)\right): A T_{z_{i}}=T_{z_{i}} A, i=1, \ldots, N\right\} .
\end{aligned}
$$

We will construct a projection onto the space of all Toeplitz operators on the $N$-torus.

THEOREM 3.1. There is a positive linear projection $\pi: B\left(H^{2}\left(\mathbb{T}^{N}\right)\right) \rightarrow$ $\mathcal{T}\left(\mathbb{T}^{N}\right)$ such that

(a) $\pi(I)=I,\|\pi\|=1$,

(b) $\pi(T)=T$ for $T \in \mathcal{T}\left(\mathbb{T}^{N}\right)$,

(c) $\pi\left(A T_{\varphi}\right)=\pi(A) T_{\varphi}$ for $A \in B\left(H^{2}\left(\mathbb{T}^{N}\right)\right)$ and $T_{\varphi} \in \mathcal{A}\left(\mathbb{T}^{N}\right)$,

(d) $\pi(A)$ belongs to the weakly-closed convex hull of $\left\{T_{z^{k}}^{*} A T_{z^{k}}: k \in \mathbb{N}^{N}\right\}$ for $A \in B\left(H^{2}\left(\mathbb{T}^{N}\right)\right)$,

(e) $\pi\left(P_{k}\right)=1$, where $P_{k}$ is the orthogonal projection on the range of $T_{z^{k}}$.

Proof. For $A \in B\left(H^{2}\left(\mathbb{T}^{N}\right)\right)$ and $x, y \in H^{2}\left(\mathbb{T}^{N}\right)$ we define

$$
[x, y]=\Lambda\left(\left\{\left(T_{z^{k}}^{*} A T_{z^{k}} x, y\right)\right\}_{k \in \mathbb{N}^{N}}\right),
$$

where $\Lambda$ denotes the multi-variable Banach limit given in Theorem 2.1. Since $(x, y) \mapsto[x, y]$ is a bounded sesquilinear form, there is an operator $\pi(A) \in B\left(H^{2}\left(\mathbb{T}^{N}\right)\right)$ such that

$$
(\pi(A) x, y)=\Lambda\left(\left\{\left(T_{z^{k}}^{*} A T_{z^{k}} x, y\right)\right\}_{k \in \mathbb{N}^{N}}\right) .
$$

From the definition it is easy to see that $\pi(I)=I$. Note that for any $i$, by Theorem 2.1(d),

$$
\begin{aligned}
\left(T_{z_{i}}^{*} \pi(A) T_{z_{i}} x, y\right) & =\left(\pi(A) T_{z_{i}} x, T_{z_{i}} y\right)=\Lambda\left(\left\{\left(T_{z^{k}}^{*} A T_{z^{k}} T_{z_{i}} x, T_{z_{i}} y\right)\right\}_{k \in \mathbb{N}^{N}}\right) \\
& =\Lambda\left(\left\{\left(T_{z^{k+e_{i}}}^{*} A T_{z^{k+e_{i}}} x, y\right)\right\}_{k \in \mathbb{N}^{N}}\right) \\
& =\Lambda\left(\left\{\left(T_{z^{k}}^{*} A T_{z^{k}} x, y\right)\right\}_{k \in \mathbb{N}^{N}}\right)=(\pi(A) x, y) .
\end{aligned}
$$

Thus $T_{z_{1}}^{*} \pi(A) T_{z_{1}}=\pi(A)$ and, by the characterization (4) of Toeplitz operators, we see that $\pi(A) \in \mathcal{T}\left(\mathbb{T}^{N}\right)$.

If $A \in \mathcal{T}\left(\mathbb{T}^{N}\right)$ then, by (4), $\left\{\left(T_{z^{k}}^{*} A T_{z^{k}} x, y\right)\right\}_{k \in \mathbb{N}^{N}}=\{(A x, y)\}_{k \in \mathbb{N}^{N}}$ is a constant sequence for all $x, y$ and $(\pi(A) x, y)=(A x, y)$ by Theorem 2.1(b), thus $\pi(A)=A$.

Formula (6) also implies that $\pi$ is positive. If (d) is not satisfied then for a given operator $A \in B\left(H^{2}\left(\mathbb{T}^{N}\right)\right)$ there are $x, y \in H^{2}\left(\mathbb{T}^{N}\right)$ such that $(\pi(A) x, y) \neq 0$, but $(B x, y)=0$ for all $B$ in the weakly-closed convex hull of $\left\{\left(T_{z^{k}}^{*} A T_{z^{k}} x, y\right): k \in \mathbb{N}^{N}\right\}$. This contradicts Theorem 2.1(c). The remaining properties follow from formula (6). 
4. 2-hyperreflexivity of Toeplitz operators on the $N$-torus. As before, for a given complex separable Hilbert space $\mathcal{H}$ we denote by $B(\mathcal{H})$ the algebra of all bounded linear operators on $\mathcal{H}$. It is well known that the space of trace class operators $\tau c$ is predual to $B(\mathcal{H})$ with the dual action $\langle A, f\rangle=\operatorname{tr}(A f)$ for $A \in B(\mathcal{H})$ and $f \in \tau c$. The trace norm in $\tau c$ will be denoted by $\|\cdot\|_{1}$. Denote by $F_{k}$ the set of operators of rank at most $k$. Every rank-one operator may be written as $x \otimes y$ for some $x, y \in \mathcal{H}$, where $(x \otimes y) z=(z, y) x$ for $z \in \mathcal{H}$. Moreover, $\operatorname{tr}(T(x \otimes y))=(T x, y)$.

Let $\mathcal{S} \subset B(\mathcal{H})$ be a norm-closed subspace. We denote by $d(T, \mathcal{S})$ the standard distance from an operator $T$ to the subspace $\mathcal{S}$. It is known that when $\mathcal{S}$ is weak ${ }^{*}$-closed, $d(T, \mathcal{S})=\sup \left\{|\operatorname{tr}(T f)|: f \in \mathcal{S}_{\perp},\|f\|_{1} \leq 1\right\}$, where $\mathcal{S}_{\perp}$ denotes the preannihilator of $\mathcal{S}$.

Recall that if $\mathcal{S}$ is a weak*-closed subspace of $B(\mathcal{H})$, then $\mathcal{S}$ is reflexive if and only if $\mathcal{S}_{\perp}$ is a closed linear span of rank-one operators contained in $\mathcal{S}_{\perp}$ (i.e., $\mathcal{S}_{\perp}=\operatorname{span}\left(\mathcal{S}_{\perp} \cap F_{1}\right)$ ). At the other extreme, if $\mathcal{S}_{\perp} \cap F_{1}=\{0\}$ then we call $\mathcal{S}$ transitive. A weak ${ }^{*}$-closed subspace $\mathcal{S} \subset B(\mathcal{H})$ is called $k$-reflexive if $\mathcal{S}_{\perp}=\operatorname{span}\left(\mathcal{S}_{\perp} \cap F_{k}\right)$. We also have a stronger property: $\mathcal{S}$ is called hyperreflexive if there is a constant $a$ such that

$$
d(T, \mathcal{S}) \leq a \sup \left\{|\langle T, x \otimes y\rangle|: x \otimes y \in \mathcal{S}_{\perp},\|x \otimes y\|_{1} \leq 1\right\}
$$

for all $T \in B(\mathcal{H})$, and $k$-hyperreflexive if there is a such that for any $T \in$ $B(\mathcal{H})$

$$
d(T, \mathcal{S}) \leq a \sup \left\{|\operatorname{tr}(T f)|: f \in \mathcal{S}_{\perp} \cap F_{k},\|f\|_{1} \leq 1\right\}
$$

The distance on the right hand side will be denoted by $\alpha_{k}(T, \mathcal{S})$. Let $\kappa_{k}(\mathcal{S})$ be the infimum of the constants $a$ in (8); we call it the $k$-hyperreflexivity constant. For further properties of $k$-reflexivity and $k$-hyperreflexivity the reader is referred to [3] and [7].

Analyzing the spaces of all Toeplitz operators on the unit circle $\mathcal{T}(\mathbb{T})$ and on the $N$-torus $\mathcal{T}\left(\mathbb{T}^{N}\right)$ from the reflexivity point of view, note first that the characterizations (2) and (4) allow us to see that both spaces are weak ${ }^{*}$-closed. The space $\mathcal{T}(\mathbb{T})$ is transitive, but 2 -reflexive (see [2]) and even 2-hyperreflexive (see [7]).

In [9] it was shown that $\mathcal{T}\left(\mathbb{T}^{N}\right)$ is transitive, thus not reflexive, but that it is 2-reflexive. Now we will show the stronger condition: 2-hyperreflexivity.

THEOREM 4.1. The space of all Toeplitz operators on the torus $\mathcal{T}\left(\mathbb{T}^{N}\right)$ is 2-hyperreflexive and $\kappa_{2}\left(\mathcal{T}\left(\mathbb{T}^{N}\right)\right) \leq 2$.

Proof. Let $A \in B\left(H^{2}\left(\mathbb{T}^{N}\right)\right)$. Since $\pi(A)$ belongs to the weakly-closed convex hull of the set $\left\{T_{z^{k}}^{*} A T_{z^{k}}: k \in \mathbb{N}^{N}\right\}$, we have 


$$
\begin{aligned}
d(A, & \left.\mathcal{T}\left(\mathbb{T}^{N}\right)\right) \leq\|A-\pi(A)\| \leq \sup _{k \in \mathbb{N}^{N}}\left\|A-T_{z^{k}}^{*} A T_{z^{k}}\right\| \\
& \leq \sup _{k \in \mathbb{N}^{N}} \sup \left\{\left|\left(\left(A-T_{z^{k}}^{*} A T_{z^{k}}\right) x, y\right)\right|: x, y \in H^{2}\left(\mathbb{T}^{N}\right),\|x \otimes y\|_{1}=1\right\} \\
& \leq \sup _{k \in \mathbb{N}^{N}} \sup \left\{\left|(A x, y)-\left(A z^{k} x, z^{k} y\right)\right|: x, y \in H^{2}\left(\mathbb{T}^{N}\right),\|x \otimes y\|_{1}=1\right\} \\
& \leq \sup _{k \in \mathbb{N}^{N}} \sup \left\{\left|\operatorname{tr}\left(A\left(x \otimes y-z^{k} x \otimes z^{k} y\right)\right)\right|: x, y \in H^{2}\left(\mathbb{T}^{N}\right),\|x \otimes y\|_{1}=1\right\} .
\end{aligned}
$$

Since $\operatorname{rank}\left(x \otimes y-z^{k} x \otimes z^{k} y\right) \leq 2$ and $\left\|x \otimes y-z^{k} x \otimes z^{k} y\right\|_{1} \leq 2$ if $\|x \otimes y\|_{1}=1$, it follows that $d\left(A, \mathcal{T}\left(\mathbb{T}^{N}\right)\right) \leq 2 \alpha_{2}\left(A, \mathcal{T}\left(\mathbb{T}^{N}\right)\right)$.

5. Projection onto Toeplitz operators on the unit ball. Let $\mathbb{B}_{N}$ be the unit ball in $\mathbb{C}^{N}$ and denote by $\sigma$ the normalized surface measure on the unit sphere $\partial \mathbb{B}_{N}$. We set $L^{2}\left(\partial \mathbb{B}_{N}\right)=L^{2}\left(\partial \mathbb{B}_{N}, \sigma\right)$ and $L^{\infty}\left(\partial \mathbb{B}_{N}\right)=$ $L^{\infty}\left(\partial \mathbb{B}_{N}, \sigma\right)$ and denote by $H^{2}\left(\partial \mathbb{B}_{N}\right), P_{H^{2}\left(\partial \mathbb{B}_{N}\right)}$ etc. the corresponding spaces and operators on $\partial \mathbb{B}_{N}$. Also the symbols $T_{z_{i}}$ and $T_{z^{k}}$ have the same meaning as before.

In [5] it was shown that

$$
\begin{aligned}
& \mathcal{T}\left(\partial \mathbb{B}_{N}\right)=\left\{A \in B\left(H^{2}\left(\partial \mathbb{B}_{N}\right)\right): A=\sum_{i=1}^{N} T_{z_{i}}^{*} A T_{z_{i}}\right\}, \\
& \mathcal{A}\left(\partial \mathbb{B}_{N}\right)=\left\{A \in B\left(H^{2}\left(\partial \mathbb{B}_{N}\right)\right): A T_{z_{i}}=T_{z_{i}} A, i=1, \ldots, N\right\} .
\end{aligned}
$$

For a given operator $A \in B\left(H^{2}\left(\partial \mathbb{B}_{N}\right)\right)$ we define by induction a sequence $\left\{A^{(n)}\right\}_{n \in \mathbb{N}}$ in $B\left(H^{2}\left(\partial \mathbb{B}_{N}\right)\right)$ :

$$
A^{(0)}=A, \quad A^{(n+1)}=\sum_{i=1}^{N} T_{z_{i}}^{*} A^{(n)} T_{z_{i}} .
$$

Note that $I^{(n)}=I$ and if $T \in \mathcal{T}\left(\partial \mathbb{B}_{N}\right)$, then $T^{(n)}=T$ by (9). Moreover, by (10),

$$
\left(A T_{\varphi}\right)^{(n)}=A^{(n)} T_{\varphi} \quad \text { for } A \in B\left(H^{2}\left(\partial \mathbb{B}_{N}\right)\right) \text { and } T_{\varphi} \in \mathcal{A}\left(\partial \mathbb{B}_{N}\right) .
$$

Lemma 5.1. If $A \in B\left(H^{2}\left(\partial \mathbb{B}_{N}\right)\right)$, then $\left\|A^{(n)}\right\| \leq 2\|A\|$.

Proof. For $k=\left(k_{1}, \ldots, k_{N}\right) \in \mathbb{N}^{N}$ we write $k !=k_{1} ! \cdots k_{N}$ ! and $|k|=$ $k_{1}+\cdots+k_{N}$. One can easily note that

$$
A^{(n)}=\sum_{|k|=n} \frac{n !}{k !} T_{z^{k}}^{*} A T_{z^{k}} .
$$

If $x \in H^{2}\left(\partial \mathbb{B}_{N}\right)$, then

$$
\left|\left(A^{(n)} x, x\right)\right| \leq \sum_{|k|=n} \frac{n !}{k !}\left|\left(A z^{k} x, z^{k} x\right)\right| \leq\|A\| \sum_{|k|=n} \frac{n !}{k !}\left\|z^{k} x\right\|^{2}
$$




$$
\begin{aligned}
& =\|A\| \sum_{|k|=n} \frac{n !}{k !} \int_{\partial \mathbb{B}_{N}}\left|z^{k} x(z)\right|^{2} d \sigma(z)=\|A\| \int_{\partial \mathbb{B}_{N}}|x(z)|^{2} \sum_{|k|=n} \frac{n !}{k !}\left|z^{k}\right|^{2} d \sigma(z) \\
& =\|A\| \int_{\partial \mathbb{B}_{N}}|x(z)|^{2}\left(\left|z_{1}\right|^{2}+\cdots+\left|z_{n}\right|^{2}\right)^{n} d \sigma(z)=\|A\|\|x\|^{2} .
\end{aligned}
$$

Thus the numerical range satisfies $w\left(A^{(n)}\right) \leq\|A\|$ and $\left\|A^{(n)}\right\| \leq 2\|A\|$ by $[6]$.

THEOREM 5.2. There is a positive linear projection $\pi: B\left(H^{2}\left(\partial \mathbb{B}_{N}\right)\right) \rightarrow$ $\mathcal{T}\left(\partial \mathbb{B}_{N}\right)$ such that

(a) $\pi(I)=I,\|\pi\| \leq 2$,

(b) $\pi(T)=T$ for $T \in \mathcal{T}\left(\partial \mathbb{B}_{N}\right)$,

(c) $\pi\left(A T_{\varphi}\right)=\pi(A) T_{\varphi}$ for $A \in B\left(H^{2}\left(\partial \mathbb{B}_{N}\right)\right)$ and $T_{\varphi} \in \mathcal{A}\left(\partial \mathbb{B}_{N}\right)$,

(d) $\pi(A)$ belongs to the weakly-closed convex hull of $\left\{A^{(n)}: n \in \mathbb{N}\right\}$ for $A \in B\left(H^{2}\left(\partial \mathbb{B}_{N}\right)\right)$.

Proof. For $A \in B\left(H^{2}\left(\partial \mathbb{B}_{N}\right)\right)$ and $x, y \in H^{2}\left(\partial \mathbb{B}_{N}\right)$ we define

$$
[x, y]=\Lambda\left(\left\{\left(A^{(n)} x, y\right)\right\}_{n \in \mathbb{N}}\right)
$$

where $\Lambda$ denotes the one-dimensional Banach limit (see Theorem 2.1). Note that $\left\{\left(A^{(n)} x, y\right)\right\}_{n \in \mathbb{N}} \in \ell^{\infty}(\mathbb{N}, \mathbb{C})$ by Lemma 5.1. Since $(x, y) \mapsto[x, y]$ is a bounded sesquilinear form, there is an operator $\pi(A) \in B\left(H^{2}\left(\partial \mathbb{B}_{N}\right)\right)$ such that

$$
(\pi(A) x, y)=\Lambda\left(\left\{\left(A^{(n)} x, y\right)\right\}_{n \in \mathbb{N}}\right) .
$$

Since $I^{(n)}=I$, we have $\pi(I)=I$.

Now, by Theorem 2.1(d), we get

$$
\begin{gathered}
\left(\sum_{i=1}^{N} T_{z_{i}}^{*} \pi(A) T_{z_{i}} x, y\right)=\sum_{i=1}^{N}\left(\pi(A) z_{i} x, z_{i} y\right) \\
=\sum_{i=1}^{N} \Lambda\left(\left\{\left(A^{(n)} z_{i} x, z_{i} y\right)\right\}_{n \in \mathbb{N}}\right)=\Lambda\left(\left\{\left(\sum_{i=1}^{N} T_{z_{i}}^{*} A^{(n)} T_{z_{i}} x, y\right)\right\}_{n \in \mathbb{N}}\right) \\
=\Lambda\left(\left\{\left(A^{(n+1)} x, y\right)\right\}_{n \in \mathbb{N}}\right)=(\pi(A) x, y) .
\end{gathered}
$$

Thus $\pi(A) \in \mathcal{T}\left(\partial \mathbb{B}_{N}\right)$ by $(9)$.

If $A \in \mathcal{T}\left(\partial \mathbb{B}_{N}\right)$ then $A^{(n)}=A$ for all $n$, and thus $\pi(A)=A$. Property (c) is a consequence of (12), and the proof of (d) is similar to that of Theorem $3.1(\mathrm{~d})$.

6. Projection onto generalized Toeplitz operators. The idea of generalized Toeplitz operators is to replace in the characterization (2) the backward shift $T_{z}^{*}$ by any contraction. Precisely, for given contractions $S, T$ 
in $B(\mathcal{H})$, an operator $X \in B(\mathcal{H})$ is called a generalized Toeplitz operator with respect to $S$ and $T$ if $X=S X T^{*}$. These operators were investigated in [11]. The space of all such operators is denoted by $\mathcal{T}(S, T)$. Note that this definition implies weak*-closedness of $\mathcal{T}(S, T)$.

In [10] this idea was extended to two variables. It is easy to extend it to the multi-variable case. Having in mind the characterization (4) of Toeplitz operators on the $N$-torus we make the following definition. For given $N$-tuples $\mathbf{S}=\left(S_{1}, \ldots, S_{N}\right)$ and $\mathbf{T}=\left(T_{1}, \ldots, T_{N}\right)$ of commuting contractions on $\mathcal{H}$, an operator $X \in B(\mathcal{H})$ is called a generalized Toeplitz operator with respect to $\mathbf{S}$ and $\mathbf{T}$ if $X=S_{i} X T_{i}^{*}$ for $i=1, \ldots, N$. The space of all such operators is denoted by $\mathcal{T}(\mathbf{S}, \mathbf{T})$. It is also weak*-closed. For a given commuting $N$-tuple $\mathbf{S}=\left(S_{1}, \ldots, S_{N}\right)$ we set $\mathbf{S}^{k}=S_{1}^{k_{1}} \cdots S_{N}^{k_{N}}$ for $k=\left(k_{1}, \ldots, k_{N}\right) \in \mathbb{N}^{N}$.

Now we extend the definition of the projection considered in Section 3 to generalized Toeplitz operators. We formulate the theorem for arbitrary $N$, but even the case $N=1$ is worth noting.

Theorem 6.1. Let $\mathbf{S}$ and $\mathbf{T}$ be two $N$-tuples of commuting contractions on $\mathcal{H}$. There is a linear projection $\pi: B(\mathcal{H}) \rightarrow \mathcal{T}(\mathbf{S}, \mathbf{T})$ such that

(a) $\|\pi\| \leq 1$,

(b) $\pi(X)=X$ for $X \in \mathcal{T}(\mathbf{S}, \mathbf{T})$,

(c) if $A \in B(\mathcal{H})$ then $\pi(A)$ belongs to the weakly-closed convex hull of $\left\{\mathbf{S}^{k} A \mathbf{T}^{* k}: k \in \mathbb{N}^{N}\right\}$.

Proof. Let $\Lambda$ be the functional from Theorem 2.1. For $A \in B(\mathcal{H})$ and $x, y \in \mathcal{H}$ we define

$$
(\pi(A) x, y)=\Lambda\left(\left\{\left(\mathbf{S}^{k} A \mathbf{T}^{* k} x, y\right)\right\}_{k \in \mathbb{N}^{N}}\right) .
$$

To check the details, one can follow the proof of Theorem 3.1.

7. 2-hyperreflexivity of generalized Toeplitz operators. The reflexive behavior of the space $\mathcal{T}(S, T)$ of generalized Toeplitz operators depends on the contractions $S, T$. For example if the underlying Hilbert space is the Hardy space on the unit circle and $S=T=T_{z}^{*}$ then $\mathcal{T}\left(T_{z}^{*}, T_{z}^{*}\right)=\mathcal{T}(\mathbb{T})$ is transitive. On the other hand, the space $\mathcal{T}(S, T)$ might be even (hyper)reflexive. For example, if $S=T=I_{H}$ then $\mathcal{T}\left(I_{H}, I_{H}\right)=B(\mathcal{H})$, which is (hyper)reflexive. However, we can estimate the reflexive behavior even for arbitrary $N$ by

TheOREM 7.1. Let $\mathbf{S}$ and $\mathbf{T}$ be two $N$-tuples of commuting contractions on $\mathcal{H}$. Then $\mathcal{T}(\mathbf{S}, \mathbf{T})$ is 2-hyperreflexive.

Proof. By Theorem 6.1(c), for any $A \in B(\mathcal{H}), \pi(A)$ belongs to the weakly-closed convex hull of $\left\{\mathbf{S}^{k} A \mathbf{T}^{* k}: k \in \mathbb{N}^{N}\right\}$. As in the proof of Theorem 
4.1 we can show that

$$
\begin{aligned}
d(A, \mathcal{T}(\mathbf{S}, \mathbf{T})) & \leq\|A-\pi(A)\| \leq \sup _{k \in \mathbb{N}^{N}}\left\|A-\mathbf{S}^{k} A \mathbf{T}^{* k}\right\| \\
& \leq \sup _{k \in \mathbb{N}^{k}} \sup \left\{\left|\operatorname{tr}\left(A\left(x \otimes y-\mathbf{T}^{* k} x \otimes \mathbf{S}^{* k} y\right)\right)\right|:\|x \otimes y\|_{1}=1\right\} .
\end{aligned}
$$

Since $\operatorname{rank}\left(x \otimes y-\mathbf{T}^{* k} x \otimes \mathbf{S}^{* k} y\right) \leq 2$ and $\left\|x \otimes y-\mathbf{T}^{* k} x \otimes \mathbf{S}^{* k} y\right\|_{1} \leq 2$ for $\|x \otimes y\|_{1}=1$, we have

$$
d(A, \mathcal{T}(\mathbf{S}, \mathbf{T})) \leq 2 \alpha_{2}(A, \mathcal{T}(\mathbf{S}, \mathbf{T})) .
$$

Theorem 7.1 for $N=1$ is also a consequence of [8].

Added in proof. D. Timotin (private communication) has shown that the norm of the projection in Theorem 5.2 is equal to $1,\|\pi\|=1$.

\section{References}

[1] W. T. Arveson, Interpolation problems in nest algebras, J. Funct. Anal. 20 (1975), 208-233.

[2] E. A. Azoff and M. Ptak, A dichotomy for linear spaces of Toeplitz operators, J. Funct. Anal. 156 (1998), 411-428.

[3] J. B. Conway, A Course in Operator Theory, Amer. Math. Soc., Providence, 2000.

[4] -, A Course of Functional Analysis, Springer, New York, 1990.

[5] A. M. Davie and N. P. Jewell, Toeplitz operators in several complex variables, J. Funct. Anal. 26 (1977), 356-368.

[6] P. R. Halmos, A Hilbert Space Problem Book, Van Nostrand, 1967.

[7] K. Kliś and M. Ptak, k-hyperreflexive subspaces, Houston J. Math., to appear.

[8] H. S. Mustafayev, On hypereflexivity of some operator spaces, Int. J. Math. Math. Sci. 19 (1996), 603-606.

[9] M. Ptak, On the reflexivity of multigenerator algebras, Dissertationes Math. 378 (1998).

[10] -, Two variable Toeplitz type operators, Acta Sci. Math. (Szeged), 71 (2005), 353362.

[11] V. Pták and P. Vrbová, Operators of Toeplitz and Hankel type, ibid. 52 (1988), 117-140.

Institute of Mathematics

University of Agriculture

Al. Mickiewicza 24/28

30-059 Kraków, Poland

E-mail:rmptak@cyf-kr.edu.pl 